\title{
Rapid detection and genotyping of ALK fusion variants by adapter multiplex PCR and high-resolution melting analysis
}

\author{
Mei $\mathrm{Li}^{1} \cdot$ Shen $\mathrm{Lu}^{2} \cdot \mathrm{Xu} \mathrm{Sun}{ }^{3}$
}

Received: 28 May 2019 / Revised: 8 August 2019 / Accepted: 7 September 2019 / Published online: 22 October 2019

(c) The Author(s), under exclusive licence to United States and Canadian Academy of Pathology 2019

\begin{abstract}
Anaplastic lymphoma kinase (ALK) fusion is a promising predictive biomarker of ALK-tyrosine kinase inhibitor (ALKTKI) treatment. Furthermore, different fusion variants correlate to different ALK-TKIs responses. Although variant identification assists in treatment direction, most ALK detection assays do not genotype different fusion variants. We developed a high-resolution melting (HRM) assay to rapidly detect ALK fusions and automatically distinguish at least 20 fusion variants in one tube. Adapter multiplex PCR was designed to amplify ALK fusion variants and the reference gene GAPDH. After HRM, negative derivative curves showed a low temperature GAPDH peak, and if an ALK fusion was present, a high temperature peak from the ALK segment and variably a middle temperature part associated with the fusion partner. Selected regions of the second derivative curves were analyzed to extract features $\left(\Delta \mathrm{T}_{\mathrm{m}}\right.$, PTS/ITS, $\left.\mathrm{H}_{1} / \mathrm{H}_{2}\right)$ that define two curve types (monotonic and non-monotonic). Synthetic samples of 20 ALK fusion variants were used to train a quadratic discriminate analysis model, and the accuracy was $97.06 \%$ (66/68) and 85.71\% (144/162) for monotonic and nonmonotonic variants, respectively. The limit of detection of the assay was $1 \%$. The analytical sensitivity of genotyping was 1 and 5\% for monotonic and non-monotonic variants, respectively. In a blinded study, we detected ALK fusion from formalin-fixed paraffin-embedded lung cancer samples with a 100\% 47) and genotyping /47) and genotyping (7/7). Multiplex adapter HRM is a simple, fast, and sensitive way of ALK fusion detection and genotyping. Automatic genotyping with parameters extracted from second derivative curves is a promising method that may be applicable to other types of gene variants detected by HRM.
\end{abstract}

\section{Introduction}

Anaplastic lymphoma kinase (ALK) fusion, caused by chromosomal rearrangement, is a driver mutation in many

Supplementary information The online version of this article (https:// doi.org/10.1038/s41374-019-0330-x) contains supplementary material, which is available to authorized users.

Mei Li

meili.path@gmail.com

$\triangle$ Shen Lu

lu.shen1956@aliyun.com

1 Scientific Research Center, The Second Hospital of Dalian Medical University, Dalian 116027, China

2 Department of Pathology, Dalian Medical University, Dalian 116044, China

3 Department of Pathology, The First Hospital of Dalian Medical University, Dalian 116011, China malignant tumors, including non-small cell lung cancer $[1,2]$. This patient subgroup can respond favorably to ALKtyrosine kinase inhibitor (ALK-TKI) treatment. However, response is variable to ALK-TKIs and only $\sim 60 \%$ have a good response [2-5]. More and more studies suggest that ALK fusion variants confer at least part of the discrepancy. ALK fusion variant genotyping may further assist in tailoring ALK-TKIs treatment [6].

ALK fusion is heterogeneous on the DNA and RNA level. Echinoderm microtubule-associated protein-like 4 (EML4), kinesin light chain 1 (KLC1), Huntingtin interacting protein 1 (HIP1), among others can break at different introns and fuse with the ALK-tyrosine kinase domain (exon 20-29) to form fusion variants [7, 8]. Detection of structural alterations is challenging, not to mention variant genotyping. The most common ALK fusion detection assays are fluorescence in situ hybridization and immunohistochemistry (IHC), which do not distinguish fusion variants $[9,10]$. Many detection assays based on reverse transcription PCR have been reported, but genotyping still 
depends on sequencing $[11,12]$. An mRNA-based assay detects ALK fusions by counting the hybridized fluorescent signals of either a junction probe or an imbalanced probe. Although cDNA synthesis and amplification are not necessary, probe design is complex and fluorescent labels are costly [13]. Moreover, specialized instrumentation is needed. Next generation sequencing (NGS) allows variant detection across the genome. With the application of new NGS strategies, such as anchored multiplex PCR enrichment, RNA based sequencing and analysis algorithm development, ALK fusion sequencing has become practical and relatively efficient [14-17]. NGS has comparable diagnostic sensitivity with IHC in ALK fusion detection, while it provides more potential therapeutic implications including ALK fusion variant identification [18-20]. However, because of complex assay design and result interpretation, NGS technology is concentrated at large academic institutions and private companies [9]. Cost, required quality assessment and management of NGS validation limit the implementation of NGS in smaller institutions [21, 22].

High-resolution melting (HRM) is a rapid, targeted gene variation detection method [23, 24]. A single nucleotide alteration changes the thermal stability of the amplicon and can be characterized by melting temperature $\left(T_{m}\right)$ and curve shape changes. Some "base-pair neutral" homozygotes and small insertion/deletions have a $T_{m}$ change close to zero, but can still be identified by using unlabeled-probes, snapback primers, or special analysis methods such as changing the melting rate, selectively using curve overlay, etc [25-28].

With the advantages of simplicity, speed, lowcontamination risks, and cost-efficiency, HRM has been widely used in detecting many kinds of gene variants including single nucleotide variations (SNVs), insertion/ deletions, methylation analysis, relative copy number variation $(\mathrm{CNV})$, small repeat sequence variants like tumor related microsatellite instability markers and short tandem repeat loci [29-32]. However, ALK fusion detection and "large fragment variants", like fusion variant discrimination has never been reported.

Generally, short PCR products with single melting peaks are favored for gene variation scanning and genotyping. However, multiple domains that result from variable GC content contain more information and can identify specific variants in longer PCR products, especially in heterogeneous tumor samples. In this study, we designed an adapter multiplex PCR amplicon with multiple domain melting curves to rapidly detect and genotype at least 20 ALK fusion variants in one tube with HRM. In addition, we introduced feature extraction of the second derivative curve to assist visual analysis to automatically discriminate ALK fusion variants.

\section{Materials and methods}

\section{Cell Lines and Tissue Specimens}

Human non-small cell lung cancer cell lines A549 and H1975 [33, 34], and H3122 and H2228 (provided by COBIOER BIOSCIENCES CO., LTD) were used as ALK fusion negative and positive samples, as well as the templates to synthesize ALK fusion variant fragments. The absence of ALK fusions in A549 and H1975, as well as ALK fusion variant 1 (E13:A20) in $\mathrm{H} 3122$ and variant 3 (E6a/b:A20) in H2228 [35], were confirmed by sequencing.

De-identified formalin-fixed and paraffin-embedded (FFPE) tissue blocks from 52 lung adenocarcinoma patients were residual tissue samples from diagnostic resections with ALK fusions by IHC staining (rabbit monoclonal D5F3, Ventana). Two or three tissue slides (10 $\mu \mathrm{m}$ thick) for each case were used for RNA extraction, which were provided blindly under the REB \#2015-29 in the Second Hospital of Dalian Medical University.

\section{RNA extraction and complementary DNA (CDNA) synthesis}

Total RNA from the four cell lines and FFPE tissues were extracted with the High Pure RNA Isolation Kit (Roche) and RNeasy ${ }^{\circledR}$ FFPE (Qiagen), respectively, according to the manufacturer's instructions. All RNA samples were quantified by the absorbance values at 260 and $280 \mathrm{~nm}$ on a NanoDrop $^{\text {TM }}$ 2000C (Thermo Scientific). Complementary DNA was synthesized with oligo-dT and random 6 mer (1:1) primed reverse transcription using PrimeScript ${ }^{\mathrm{TM}} \mathrm{II}$ 1st Strand cDNA Synthesis Kit (Takara) following the manufacturer's protocol. All cDNA samples were diluted fourfold before use unless otherwise specified.

\section{ALK fusion variant standard sample preparation}

Twenty ALK fusion variants in lung cancer, according to the COSMIC v85 database, were constructed as standards, including EML4-ALK (E2:A20, E3:A20, E6a/b:A20, E10: A20, E13:A20, E14:A20, E15:A20, E17:A20, E18:A20, E20: A20), STRN-ALK (S3:A20), TFG-ALK (T4:A20), KLC1ALK (KL9:A20, KL10:A20), HIP1-ALK (H21:A20, H28: A20, H30:A20), and KIF5B-ALK (KI15:A20, KI17:A20, KI24:A20). All ALK fusion variant fragments were synthesized with self-annealing PCR (Supplementary Fig. 1), except that two EML4-ALK variants (E6a/b:A20 and E13:A20) were amplified from $\mathrm{H} 2228$ and $\mathrm{H} 3122$, respectively. A $312 \mathrm{bps}$ fragment of glyceraldehyde-3-phosphate dehydrogenase (GAPDH) was amplified from A549 cDNA with the primers ACAGTCAGCCGCATCTTCTT and TTGATT TTGGAGGGATCTCG. All PCR products were purified with 
the MiniBEST Agarose Gel DNA Extraction Kit Ver.4.0 (TaKaRa) and quantified by absorbance at 260 and $280 \mathrm{~nm}$ on a NanoDrop ${ }^{\mathrm{TM}} 2000 \mathrm{C}$ and then converted to copy number concentrations by molecular weight. The 20 ALK fusion variant fragments were confirmed by sequencing (Supplementary Table 1). The GAPDH fragment was serially diluted tenfold to quantify GAPDH copy number in A549 cDNA. All ALK fusion variant fragments were $10^{8}-10^{9}$ folded diluted (Final concentration: about $1 \times 10^{3}$ copies $/ \mu \mathrm{L}$ ) and mixed into A549 cDNA, respectively, as ALK fusion variant standards. The copy number ratio of GAPDH to ALK fusion variant was about 5-10:1.

\section{Adapter multiplex PCR primer and PCR product design}

The adapter multiplex PCR used both adapter-primers and adapters as primers to amplify target fragments by multistep PCR. Adapter-primers combined the target gene sequence with a common adapter at the $5^{\prime}$ end. The target gene sequences flanked the break point in each ALK fusion variant, including the forward primer on a fusion partner exon and a shared reverse primer on ALK exon 20. Target gene primers were designed with Primer 3 (http://bioinfo.ut. ee/primer30.4.0/). Adapters were randomly designed with a $T_{m}$ about $15^{\circ} \mathrm{C}$ lower than the gene specific primers with no specific product by NCBI primer-BLAST using Homo sapiens Refseq mRNA. All ALK fusion amplicons were designed with the melting curve shape as variable as possible, but all with a melting peak around $90^{\circ} \mathrm{C}$. The GAPDH amplicon was designed with a single melting peak around $80^{\circ} \mathrm{C}$. The melting curve shape and $T_{m}$ values of amplicons were predicted with uMelt software (http://dna. utah.edu/umelt/umelt.html and http://dna.utah.edu/umelt/ umb.php). Primer dimers were avoided by using the Multiple Primer Analyzer (https://www.thermofisher.com). All optimized final primers are listed in Supplementary Table 2.

\section{Adapter multiplex PCR and HRM}

Adapter multiplex PCR and HRM were performed with a rotor-disk 72 well rotor on a Rotor-Gene ${ }^{\circledR} \mathrm{Q}$ (Qiagen) instrument in $10 \mu \mathrm{L}$ volumes consisting of $2 \mu \mathrm{L}$ cDNA template, $0.1 \mu \mathrm{M}$ each adapter-primer of ALK fusion variants, $0.05 \mu \mathrm{M}$ each adapter-primer of GAPDH, $0.5 \mu \mathrm{M}$ each adapter, $0.2 \mathrm{mmol} / \mathrm{L}$ each $\mathrm{dNTP}, 1 \times \mathrm{PCR}$ Buffer (with $2.5 \mathrm{mM} \mathrm{MgCl}{ }_{2}$ ), $1 \times$ LCGreen $^{\circledast}$ Plus dye (BioFire Defense), and $0.5 \mathrm{U}$ TaKaRa Taq HS with $15 \mu \mathrm{L}$ mineral oil overlay. Adapter multiplex PCR was performed with four steps: 3 cycles of $98^{\circ} \mathrm{C}, 20 \mathrm{~s}, 60^{\circ} \mathrm{C}, 20 \mathrm{~s}, 72^{\circ} \mathrm{C}, 10 \mathrm{~s}$, then 5 cycles of $98^{\circ} \mathrm{C}, 20 \mathrm{~s}, 68^{\circ} \mathrm{C}, 20 \mathrm{~s}, 72^{\circ} \mathrm{C}, 5 \mathrm{~s}$, then 10 cycles of $98^{\circ} \mathrm{C}, 20 \mathrm{~s}, 70^{\circ} \mathrm{C}, 20 \mathrm{~s}, 72^{\circ} \mathrm{C}, 5 \mathrm{~s}$, finally with 25 cycles of $98^{\circ} \mathrm{C}, 20 \mathrm{~s}, 52^{\circ} \mathrm{C}, 10 \mathrm{~s}, 72^{\circ} \mathrm{C}, 10 \mathrm{~s}$.
HRM was performed after denaturation for $30 \mathrm{~s}$ at $98^{\circ} \mathrm{C}$, by melting from $65^{\circ} \mathrm{C}$ to $97^{\circ} \mathrm{C}$ with acquisition each 0.5 degrees and other parameter defaulted. Single acquisition during PCR and HRM used the green and HRM channels, respectively $(470$ or $460 \mathrm{~nm}$ excitation and $510 \mathrm{~nm}$ emission).

\section{Agarose gel electrophoresis and sequencing}

Adapter multiplex PCR products were run on a 3\% agarose gel to test and verify the PCR specificity. All ALK fusion positive samples from 52 FFPE tissues were also electrophoresed on agarose gels and purified with MiniBEST Agarose Gel DNA Extraction Kit Ver.4.0 (TaKaRa) and Sanger sequenced with the common reverse primer (CAAAGCAGTAGTTGGGGTTGTAGTC).

\section{Analysis}

Negative derivative curves were obtained by melting curve analysis with instrument software. Fluorescent exponential background was removed and curves normalized as previously described [36]. The temperatures below and above the transition regions were identified at $77^{\circ} \mathrm{C}$ and $95^{\circ} \mathrm{C}$, respectively. The second derivative curve was calculated as $\left(F^{\prime}{ }_{n+1}-F_{n}{ }_{n}\right) /\left(T_{n+1}-T_{n}\right)$ from the negative derivative plots after background removal and normalization as detailed in Supplementary Table 3. Feature parameters were calculated manually with the data extracted from the second derivative plots. An automatic genotyping model was designed based on discriminate analysis using biotools, MASS packages in $\mathrm{R}$ software with ALK fusion variants classified according to feature parameters. The automatic genotyping model was trained with the parameters from the standards. The genotypes of FFPE and cell line samples were then assigned by the model. Means, standard deviations (SD), 95\% confidence intervals (95\% CIs), Pearson correlation analysis, Box's M tests, discriminant analysis, etc. were all performed with Microsoft Excel or R software version 3.5.2.

\section{Results}

Adapter multiplex PCR was designed to amplify ALK fusion variants and GAPDH simultaneously (Fig. 1a). Twenty ALK fusion variants were amplified. After the first cycles of amplification, the GAPDH fragment and any ALK fusion variant present is amplified by the low concentration adapter-primers $(0.05-0.1 \mu \mathrm{M})$ at a high annealing temperature $\left(68-70{ }^{\circ} \mathrm{C}\right)$. High concentration common adapters $(0.5 \mu \mathrm{M})$ anneal at a lower temperature $\left(52{ }^{\circ} \mathrm{C}\right)$ during the later cycles to complete the final amplification. The GAPDH fragment is amplified in all samples 
A

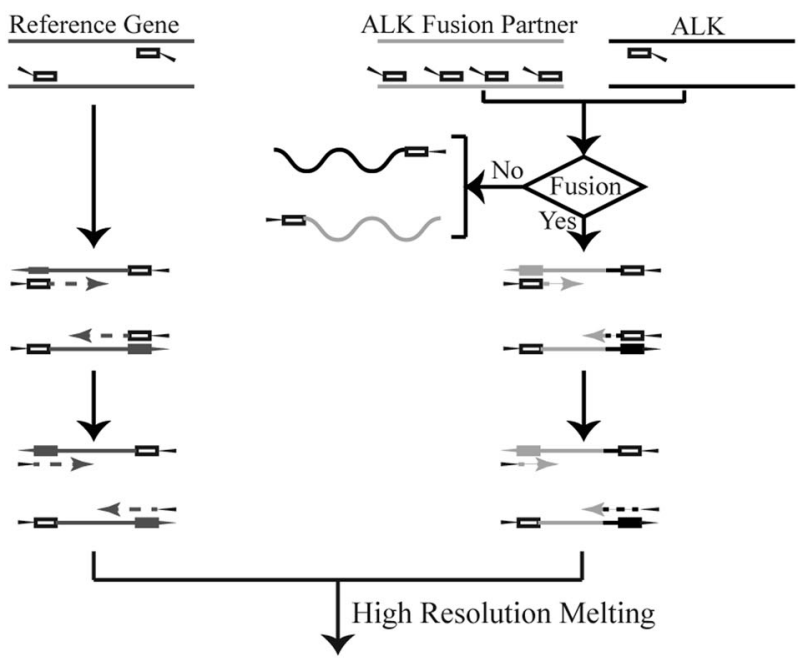

B

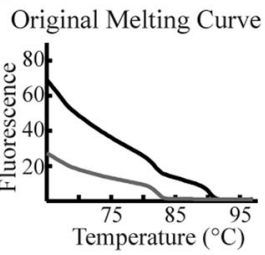

D Background Removed

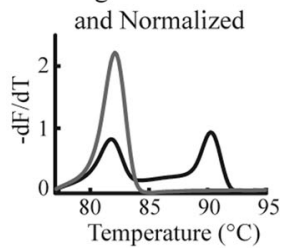

$\mathrm{C}$

Negative First Derivative

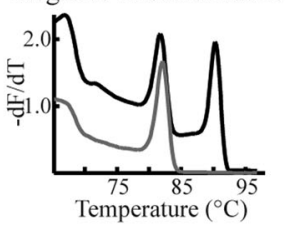

E Second Derivative

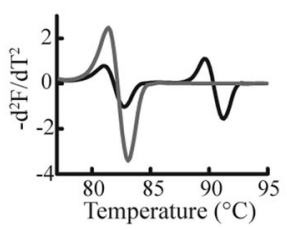

Fig. 1 Diagram of adapter multiplex PCR (a) and high-resolution melting (b-e) in ALK fusion variant detection. Adapter multiplex PCR is a multistep amplification with adapter-primers and adapters as primers. The adapter-primers include a common adapter sequence (black wedge) at the $5^{\prime}$ end of gene specific primers (empty rectangle). Adapter-primers first target and enrich gene fragments including GAPDH and, if present ALK fusion fragments at a high annealing temperature. In later cycles, common adapters complete the final amplification at a low annealing temperature. Amplicons consist of a variable fusion partner fragment, a common ALK fragment and adapters on both ends. A high-resolution melting process (b) is run after amplification. Data are extracted from melting curve analysis with the instrument software (c). After exponential background removal and normalization (d), second derivative plots are calculated (e). Melting curves in b-e showed an ALK fusion positive sample (H3122, black line) and negative sample (A549, grey line). Calculations are shown in Supplementary Table 3. ALK fusion negative samples only display the GAPDH fragment (low temperature peak), while ALK fusion positive samples display both the GAPDH peak and the ALK fusion (high temperature peak)

unless poor sample quality results in PCR failure. Best results were obtained with an adapter-primer concentration ratio of 1: 2 (GAPDH:ALK fusion variants). After HRM,

ALK fusion and variant genotypes were called by analyzing negative derivative and second derivative melting plots (Fig. 1b-e).

The ALK fusion partner fragments were of variable size (25-198 bps) and GC content (36.7-59.4\%) and were joined to a common ALK fragment (Fig. 2a, b). The common ALK fragment was 123 bps with a high GC content $(57.7 \%)$. Also common to all fusion amplicons were two 18 bps low GC content (44\%) adapters at the $5^{\prime}$ and $3^{\prime}$ ends to increase the potential number of melting domains within the amplicon. All ALK fusion variant amplicons displayed a marked high temperature melting domain mainly contributed by the common ALK fragment. The fusion variants showed a single melting domain when their size was less than 123 bps and GC content was more than $47 \%$. Otherwise, a second melting domain was present (Fig. 2c).

The PCR products of 20 ALK fusion variant standard are shown on agarose gels in Supplementary Fig. 2. The amplicon of GAPDH was $95 \mathrm{bps}$, and ALK fusion variant amplicons varied from 184 to 326 bps. Negative derivative plots and second derivative plots of the 20 ALK fusion variants are shown in Fig. 3. The GAPDH amplicon is a low temperature peak around $82{ }^{\circ} \mathrm{C}$, and all ALK fusion variants display a high temperature derivative peak around $90^{\circ} \mathrm{C}$. Six variants (KL9:A20, E18:A20, KA17:A20, H28:A20, E13:A20, E17:A20) had only one ALK fusion derivative peak, while the other variants showed two derivative peaks. With the GAPDH derivative peak as a low temperature control, fusion variants may be genotyped visually by melting curve shape and melting temperature difference $\left(\Delta T_{m}\right)$ to the GAPDH peak. The $\Delta T_{m}$ differences were very small between KL9:A20 and H28:A20 $\left(0.08^{\circ} \mathrm{C}\right)$ and E13:A20 and KA17:A20 $\left(0.097^{\circ} \mathrm{C}\right)$, but they could be distinguished by visually comparing their second derivative plots.

Considering the huge heterogeneity of clinical tumor samples, we selected key parameters from the second derivative curve for automatic genotyping by discriminant analysis. We recorded temperature and $-\mathrm{d}^{2} \mathrm{~F} / \mathrm{dT}^{2}$ values at key points (Fig. 4, point a-f) on the second derivative plots to calculate $\Delta T_{m}$, the peak temperature span (PTS), the inner temperature span (ITS), and the peak height ratio $\left(\mathrm{H}_{1} / \mathrm{H}_{2}\right)$. The six fusion variants with a single melting peak, and an internal monotonic increase in the second derivative plot at the interval $[\mathrm{b}, \mathrm{d}]$, were characterized with parameters $\Delta \mathrm{T}_{\mathrm{m}}$, PTS, $\mathrm{H}_{1} / \mathrm{H}_{2}$ (Fig. 4a), and variants with multiple melting peaks, that were not monotonic, were characterized with parameters $\Delta \mathrm{T}_{\mathrm{m}}$, ITS, $\mathrm{H}_{1} / \mathrm{H}_{2}$ (Fig. 4b). Each ALK fusion standard was amplified at least ten times to calculate the parameters and train the discriminant analysis (Supplementary Table 4). Parameters from both internal monotonic or non-monotonic plots rejected the homogeneity hypothesis of covariance matrices ( $p$ values were $6.82 \mathrm{e}-05$ and 
A

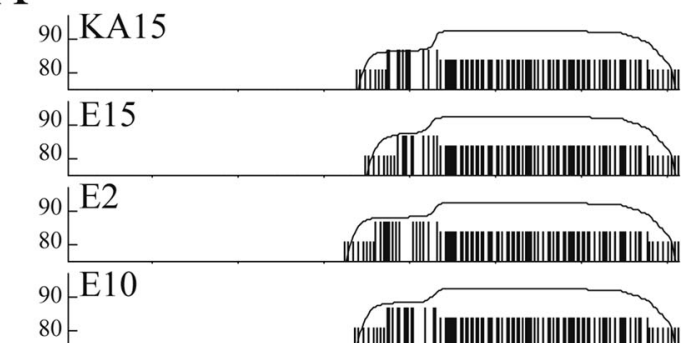

${ }_{90}+\mathrm{E} 6 \mathrm{a}$

$80-$

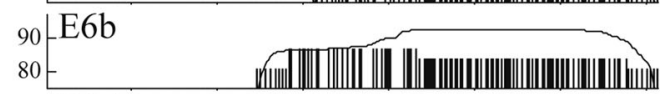

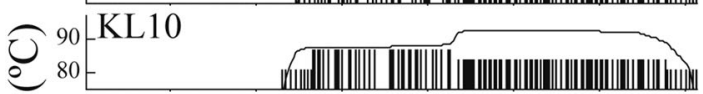

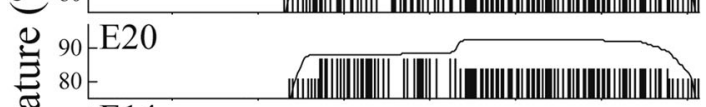

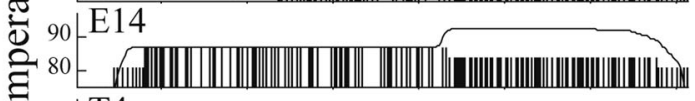

음
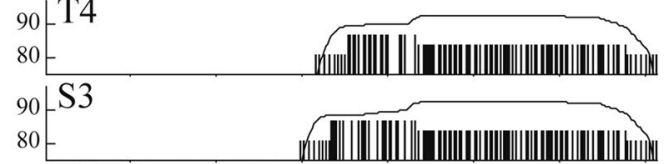

${ }_{80}{ }_{80}^{\text {KA24 }}$

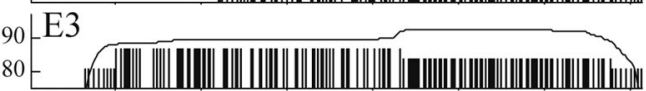
80 80- $\underbrace{\mathrm{H} 21}_{50}$

Index

Fig. 2 Characteristics of 20 ALK fusion amplicons (all fused with A20). Temperature profiles of ALK fusion variant amplicons (a, b) were generated by uMelt at medium resolution $\left(0.5^{\circ} \mathrm{C}\right.$ steps $)$ with $20 \mathrm{mM}\left[\mathrm{Mono}^{+}\right], 1.7 \mathrm{mM}$ free $\left[\mathrm{Mg}^{+}\right]$, and 0 DMSO\% (Dwight et al. 2011). The black bars indicate GC base pairs, the white bars AT base pairs. Amplicons in a show two melting domains, while those in b show a single melting domain. All amplicons include a variable

$9.398 \mathrm{e}-08$, respectively), so a quadratic discriminant analysis model was selected. The ALK fusion variants distributed between all two parameters combinations are shown in Fig. 5. Three parameters were combined to best genotype ALK fusion variants. The accuracy was $97.06 \%$ (66/68) in distinguishing monotonic variants and $85.71 \%$ $(144 / 162)$ in non-monotonic variants (Supplementary Table 5).

We blindly tested for ALK fusions in 52 FFPE samples. By comparison to the melting curves of the standard samples in Fig. 3, 7 samples were ALK fusion positive, 40 samples negative, and 5 samples without specific
B
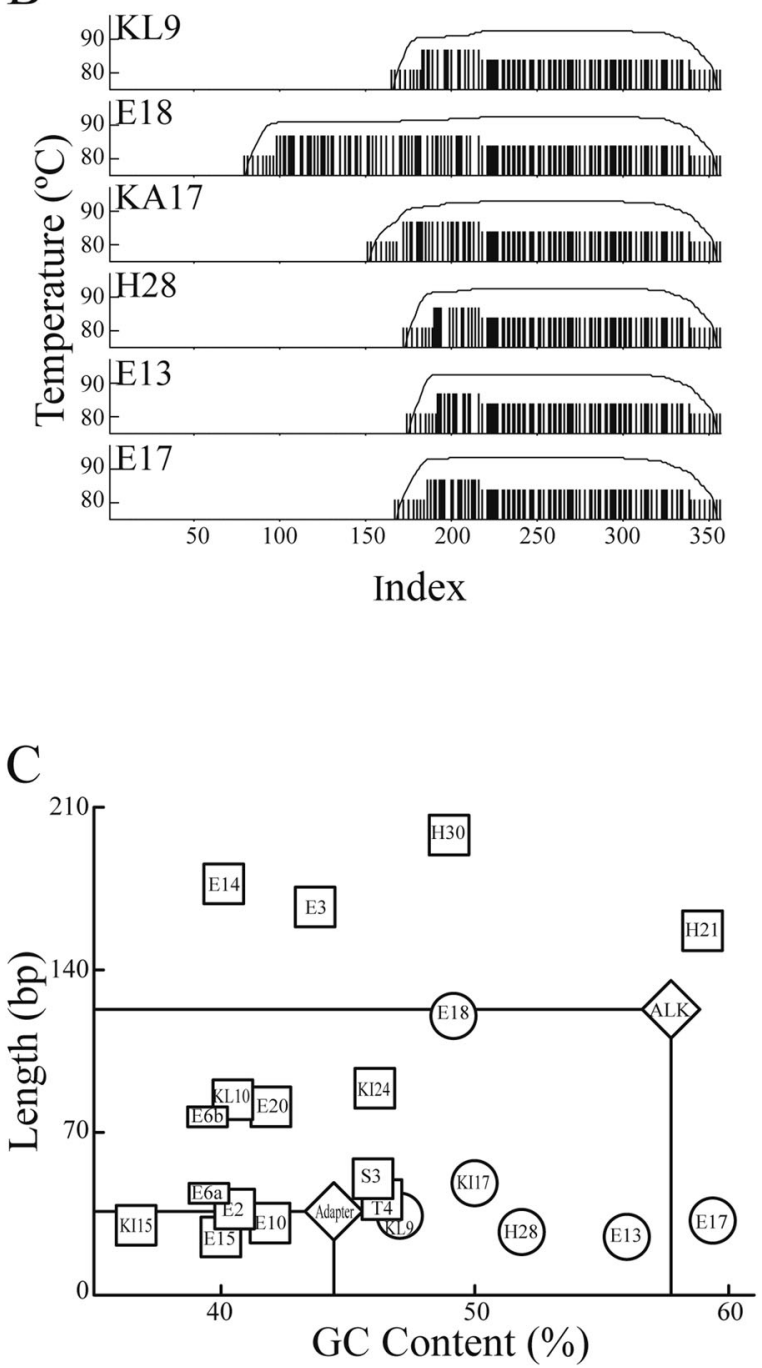

fusion partner fragment (high bars) and a common ALK exon 20 fragment (middle bars) between two adapters (low bars). c The length and GC content of variable fusion partner fragments (circles, squares, or rectangles) compared to the adapter sequence (diamond) and ALK fragment (diamond). One ALK fusion variant (E6a/b:A20) has two fusion partner fragments (rectangles)

GAPDH product. All positive and negative samples were $100 \%$ concordant with ALK IHC detection. Furthermore, multiplex PCR was able to distinguish between the different fusion variants. We plotted the feature parameters of the fusion positive FFPE samples (P1-P7) against the standard samples (Fig. 5). By both visual identification and automatic genotyping by feature extraction, the seven positive samples were identified as E6:A20 for P1 and P2, KL9:A20 for P3, and E13:A20 for P4-P7 (Supplementary Fig. 3), which were all confirmed by Sanger sequencing. ALK fusion variants were identified with $100 \%$ (7/7) accuracy by extraction of feature parameters. 
Fig. 3 Melting curves of an ALK fusion negative sample (dotted lines) and 20 synthesized ALK fusion variant standards (solid lines). The negative derivative plots (black lines) and the second derivative plots (grey lines) of all samples in triplicate are shown. In the negative derivative plots, ALK fusion negative samples only show a GAPDH melting peak around $82{ }^{\circ} \mathrm{C}$, while ALK fusion variants that all fused with ALK exon 20 show one or two other peaks, around $86-90{ }^{\circ} \mathrm{C}$. The melting curve shape of ALK variants is different among variants, as shown with negative first derivative and second derivative plots

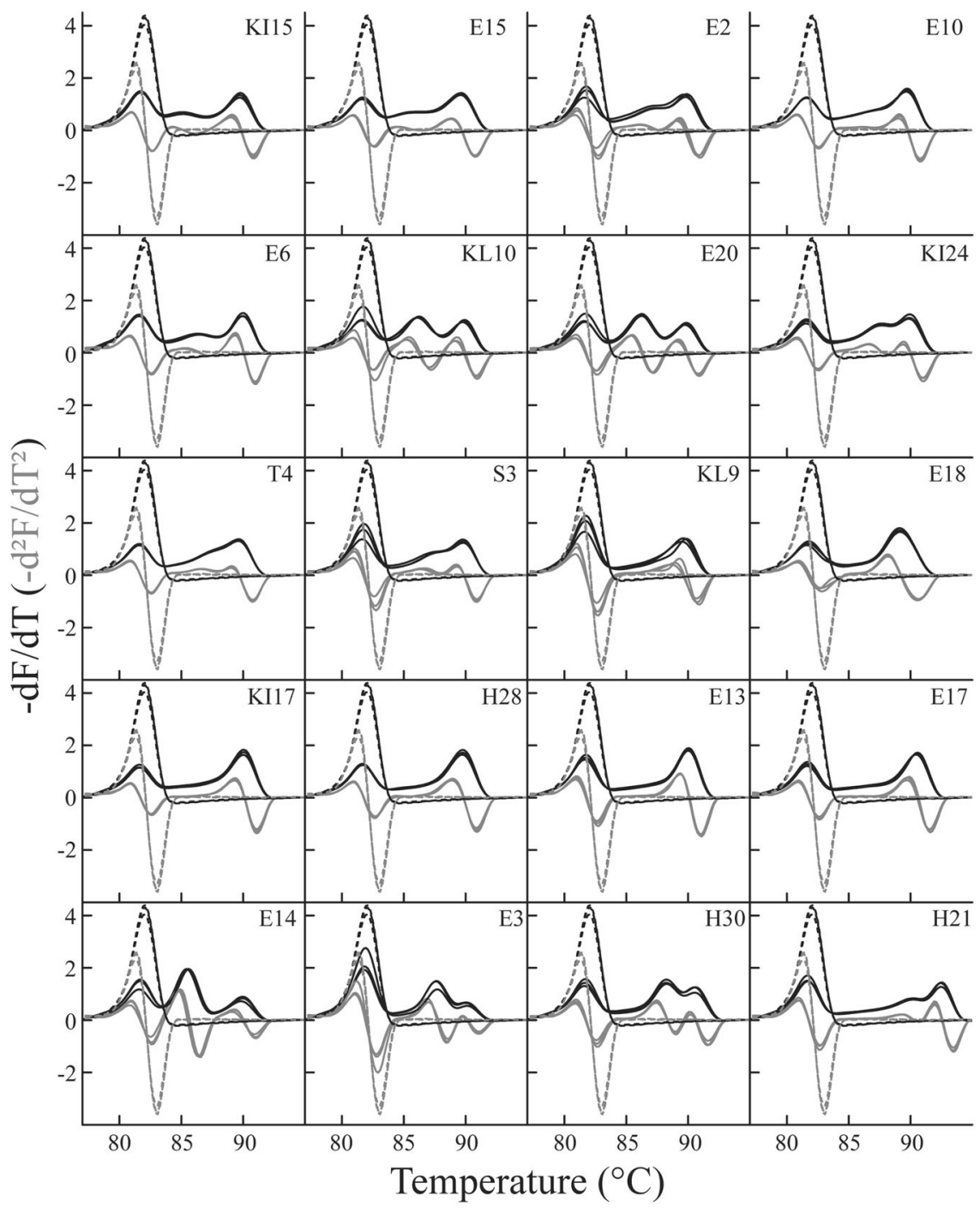

The analytical sensitivity of detection and genotyping was determined with serial fourfold dilutions of $\mathrm{H} 3122$ and $\mathrm{H} 2228$ cDNA as well as mixing $\mathrm{H} 3122$ and $\mathrm{H} 2228$ cDNA into A549 cDNA at ratios of 1\%, 2.5\%, 5\%, 10\%, 20\%, $30 \%, 40 \%, 50 \%, 60 \%, 70 \%, 80 \%, 90 \%$, and $100 \%$. Amplification plots, negative derivative plots, and the second derivative plots are shown in Fig. 6. The ALK fusion peak becomes lower as the template concentration or the positive sample proportion decreases. Visually, all dilutions and ratios were discernable, although the peaks became very small in low $\mathrm{H} 2228$ proportion samples (Fig. 6b). The analytical sensitivity of $\mathrm{H} 3122$ and $\mathrm{H} 2228$ by automatic genotyping was 1 and 5\%, respectively. Low template concentrations may lead to incorrect genotyping of H3122 but not H2228 (Supplementary Tables 6-8). Parameters $\Delta T_{m}$, PTS, and ITS were weakly affected by sample concentrations or ALK fusion proportions, while $\mathrm{H}_{1} / \mathrm{H}_{2}$ was correlated after data was logarithmically transformed (Supplementary Fig. 4 and Supplementary Table 9).

\section{Discussion}

ALK fusion has become a promising biomarker in predicting patient response to ALK-TKIs treatment. Recently, some studies, either in vivo or in vitro, have suggested that ALK fusion partners affect the fusion protein stability, kinase activity, and treatment efficacy [37]. Woo et al. reported that ALK fusion variant 3 (E6a/b:A20) showed significantly shorter progression free survival (PFS) with crizotinib, alectinib, and ceritinib treatment, and more commonly developed ALK resistant mutations, especially ALK G1202R [38-41]. Fortunately, variant 3 showed longer PFS to third-generation ALK-TKI lorlatinib treatment [41]. Variant 1 (E13:A20) not only displayed better efficacy with crizotinib, but showed longer PFS with pemetrexed [42, 43]. ALK fusion variant identification has potential clinical significance, and further study is needed. Rapid ALK detection and genotyping is important, a requirement that challenges current ALK detection methods. 

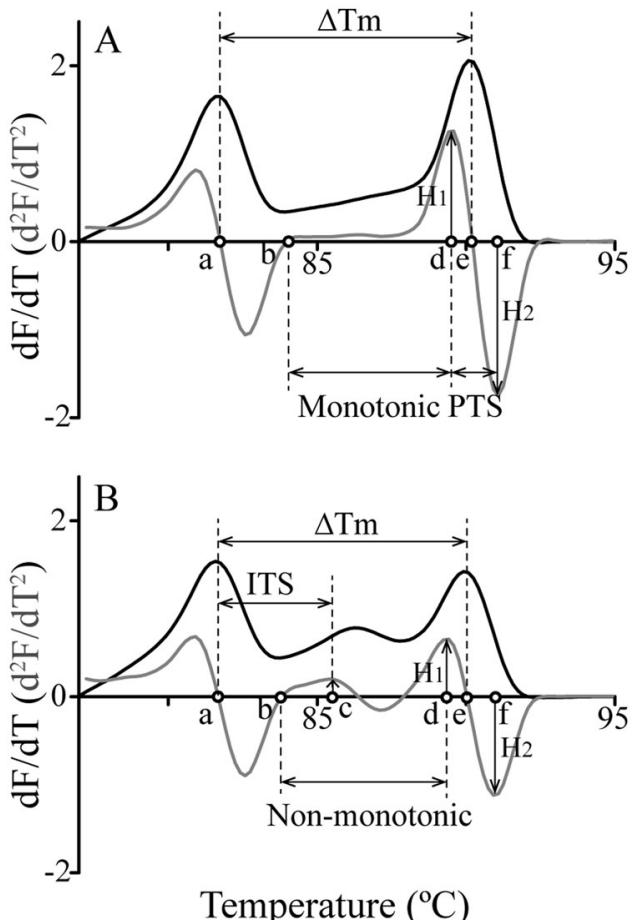

Temperature $\left({ }^{\circ} \mathrm{C}\right)$

Fig. 4 Parameters of the second derivative plots of ALK fusion variants. Some key points are marked as $\mathbf{a}$, b, e (zero point) and c, d, f (local peak point). All ALK fusion variant amplicons were divided into monotonically increasing (a) and non-monotonic (b) at the interval $[b, d]$ of second derivative plots. The negative derivative plot (black line) and the second derivative plot (grey line) of variant E13:A20 (a) and variant E6a/b:A20 (b) are shown. The interpeak temperature difference $\left(\Delta T_{m}\right)$ (interval $\left.[\mathrm{a}, \mathrm{e}]\right)$, peak temperature span (PTS) (interval $[\mathrm{d}, \mathrm{f}])$ and the peak height ratio $\left(H_{l} / H_{2}\right)$ were used as parameters for monotonically increasing melting plots. $\Delta T_{m}$, inner temperature span (ITS) (interval $[\mathrm{a}, \mathrm{c}]$ ) and $H_{l} / \mathrm{H}_{2}$ were used as parameters for non-monotonic melting plots

We developed a multiplex HRM assay to detect ALK fusions and distinguish between at least twenty different variants in one tube simultaneously. In a blinded analysis of 52 FFPE samples, 47 samples were successfully amplified and correlated perfectly to IHC results. The detection was not affected by low quantity of sample, either in low template concentration or in a low proportion of fusion RNA (Fig. 6). However, good RNA quality is necessary for specific amplification. All 52 FFPE samples were stored about 1 year at room temperature and RNA degradation led to amplification failure of five samples $(9.6 \%)$.

Usually, SNVs, small insertion/deletions and even small repeat sequence variants are distinguished well with normalized melting curves and difference curves [29, 44]. Negative derivative curves display fluorescence decreases as melting peaks. Specific genotyping of multiple variants within one or multiple amplicons is possible $[24,36]$. When large amplicons are analyzed such as the ALK fusion PCR products studied here, multiple melting domains increase the resolution of analysis. Both melting rate (first derivative)
A

B
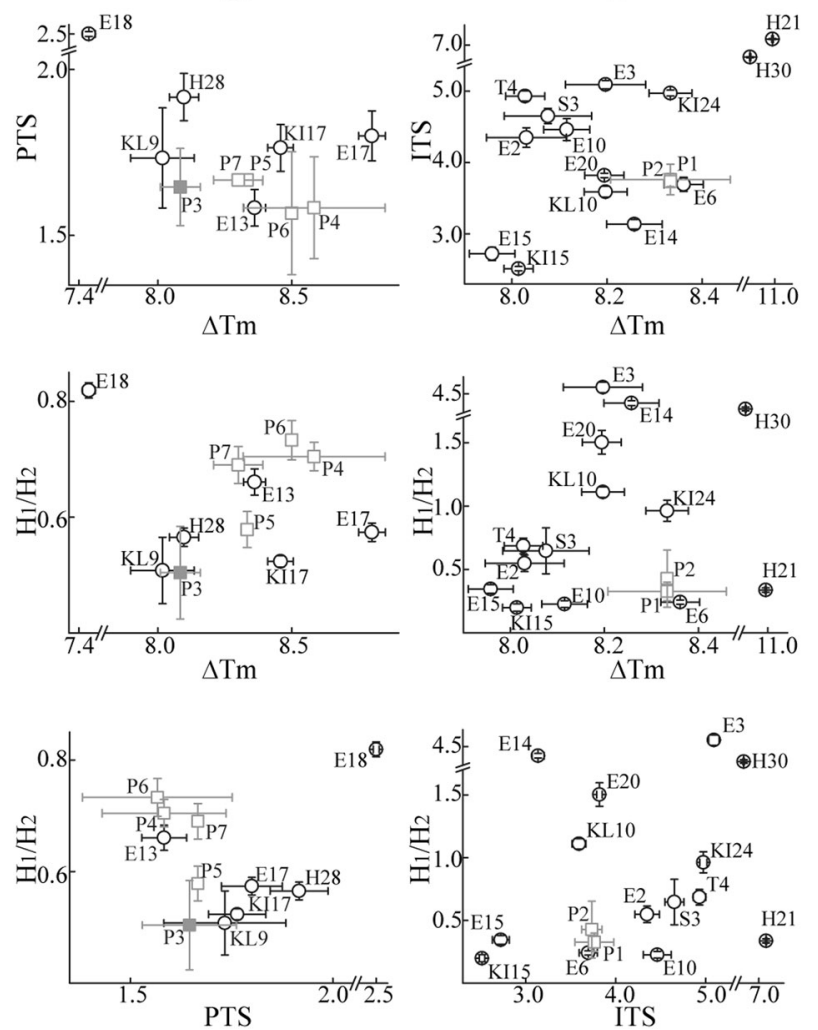

Fig. 5 ALK fusion variant distribution across any two parameters from the second derivative curve. Twenty ALK fusion variant standards (empty circles) were separated in two-dimensions, including monotonic (a) and non-monotonic variants (b). ALK fusion positive FFPE samples (P1-P7, gray solid and empty squares) are also shown. P1 and $\mathrm{P} 2$ were close to variant E6a/b:A20, P3 was close to variant KL9:A20, and P4-P7 were close to variant E13:A20. Horizontal and vertical bars indicate the $95 \%$ confidential intervals from repeated samples

and melting acceleration (second derivative) plots are informative and associated with melting domains. Parameters from these plots were extracted from the curves to enable genotyping of different fusion variants, primarily from differences in the $25-198$ bps of the fusion partner. We identified three kinds of variants in seven ALK fusion positive FFPE samples.

The parameters from second derivative curves (Fig. 4) were used for automatic genotyping with an accuracy of 91.3\% (210/230). All seven ALK fusion positive FFPE samples were genotyped with $100 \%$ accuracy when several replicates were averaged. Using individual analyses resulted in an accuracy of $89.5 \%$ (34/38). Parameters related to temperature $\left(\Delta T_{m}\right.$, PTS, ITS) were little affected by ALK fusion proportion or template concentrations, but parameter $\mathrm{H}_{1} / \mathrm{H}_{2}$, and the ratio of point $\mathrm{c}$ peak height to $\mathrm{H}_{1}$ depended on the template concentration (Supplementary Table 9). In a prior study, peak height ratio of heterozygotes from negative derivative plots was affected by melting rates [25]. 
$\mathrm{H} 2228$

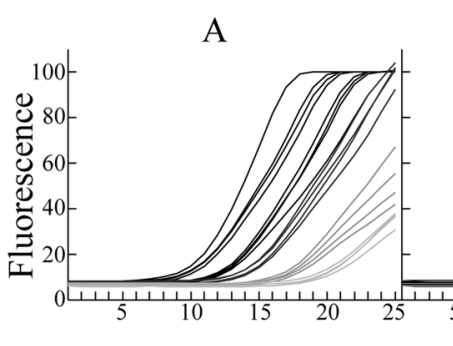

B

$\mathrm{C}$

\section{H3122}

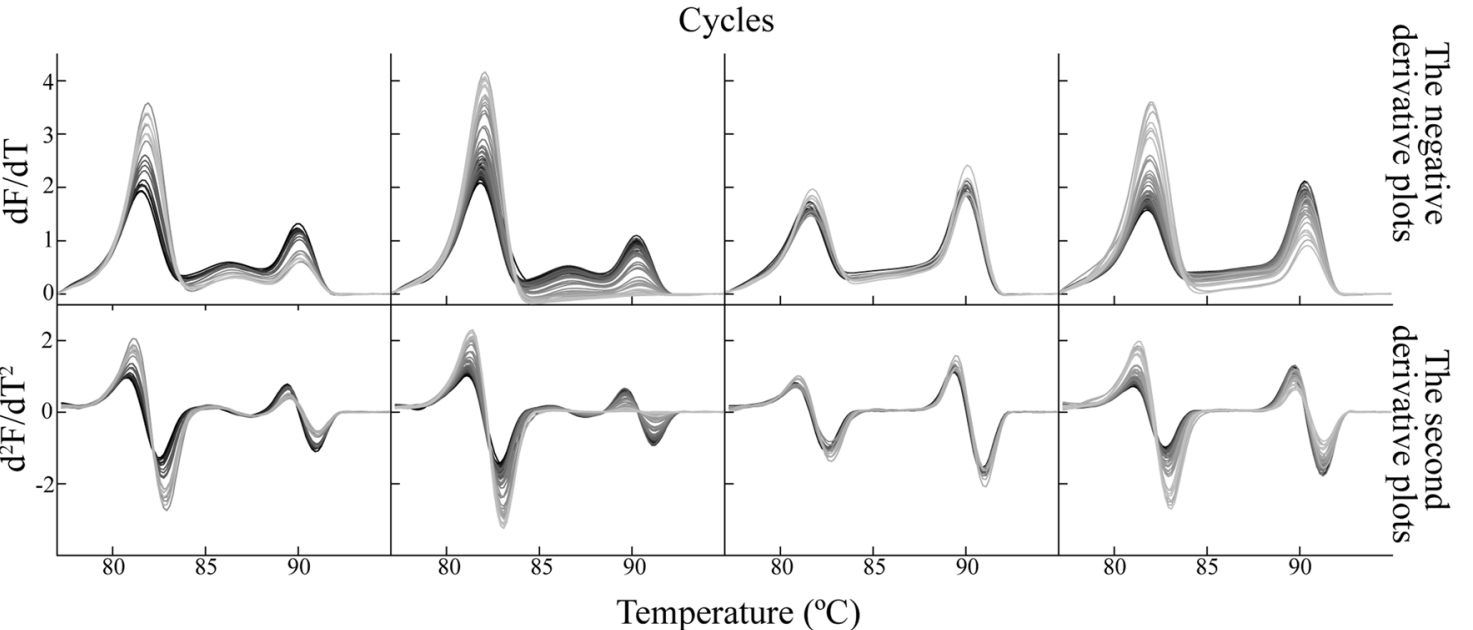

Fig. 6 The sensitivity test of ALK fusion detection and genotyping. $\mathrm{H} 3122$ and H2228 cDNA were fourfold diluted (concentration of 165 , 41.6, 10.4, 3.1, and $0.8 \mathrm{ng} / \mu \mathrm{L}$ in $\mathrm{H} 3122$ and $172.7,44.2,11.4,3.4$, $0.8 \mathrm{ng} / \mu \mathrm{L}$ in $\mathrm{H} 2228$ ) (a, c) as well as mixed into A549 cDNA with a ratio of $1 \%, 2.5 \%, 5 \%, 10 \%, 20 \%, 30 \%, 40 \%, 50 \%, 60 \%, 70 \%, 80 \%$,

The main limitation of this study is that not all ALK fusion break point were analyzed. For example, fusions with ALK exon 19 occur [45] and those with insertion sequences [46] were not analyzed. However, the method is flexible and additional breakpoints can be included as desired.

High-speed melting do not mean compromising the "resolution" of variant genotyping [25, 47]. For example, HRM for heterozygote detection on a RotorGene Q performed at $0.1^{\circ} \mathrm{C} / \mathrm{s}$ is equivalent to the default setting that is ten times slower $(0.1$ degree each step with $2 \mathrm{~s}$ holds is about $0.01{ }^{\circ} \mathrm{C} / \mathrm{s}$ ) [48]. In this study, we used a fast mode (0.5 degree each step) to do melting. After cDNA synthesis, only $79 \mathrm{~min}$ were required for ALK detection and genotyping including $75 \mathrm{~min}$ for amplification and $4 \mathrm{~min}$ for melting. With extreme PCR and high-speed melting, ALK fusion detection and genotyping might only take a few minutes [47, 49].

With more and more targeted inhibitors benefiting lung cancer patients, the number of genes to be tested also increases. At some point, massively parallel sequencing panels become more efficient than multiple single-gene tests [9]. However, cost efficient NGS typically requires large volumes and batching of multiple samples that increase
$90 \%, 100 \%$ (b, d). Every sample was repeated 4 times. Amplification plots, negative derivative plots, and second derivative plots are shown. As the H2228 or H3122 concentrations decrease, the line color goes from black, dark grey to light grey. The ALK fusion peak is smaller when the sample concentration or ALK fusion proportion is lower

turn-around times for individual patients. The overhead in instrumentation, reagents, and bioinformatics required for NGS is also in stark contrast to a single RT-PCR with HRM for ALK fusion detection and genotyping. NGS is not the only strategy to do multiplex-gene detection. HRM can also detect all types of gene alterations including SNVs, insertion/deletions, CNVs and gene fusions. Quadruplex genotyping with HRM has been reported in one tube [50]. Furthermore, multiplex-gene variant detection based on HRM only requires a few hours and individual samples can be run without batching requirements.

In summary, we have developed a multiplexed HRM assay to rapidly detect ALK fusions and automatically genotype variants. Feature extraction from the second derivative curve enables automatic genotyping, and may be useful generally in HRM analysis.

Acknowledgements This project was supported by National Natural Science Foundation of China (No. 81501832 and No. 81572919).

\section{Compliance with ethical standards}

Conflict of interest The methods in this manuscript (including the multiplex HRM design for rapid ALK fusion detection and genotyping as well as synthesis of various ALK fusion variants) form the basis of 
patent applications (201910125272.7 and 201910124977.7) in China. The authors declare that they have no conflict of interest.

Publisher's note Springer Nature remains neutral with regard to jurisdictional claims in published maps and institutional affiliations.

\section{References}

1. Li T, Kung HJ, Mack PC, Gandara DR. Genotyping and genomic profiling of non-small-cell lung cancer: implications for current and future therapies. J Clin Oncol. 2013;31:1039-49.

2. Kwak EL, Bang YJ, Camidge DR, Shaw AT, Solomon B, Maki $\mathrm{RG}$, et al. Anaplastic lymphoma kinase inhibition in non-smallcell lung cancer. N Engl J Med. 2010;363:1693-703.

3. Shaw AT, Kim DW, Nakagawa K, Seto T, Crino L, Ahn MJ, et al. Crizotinib versus chemotherapy in advanced alk-positive lung cancer. N Engl J Med. 2013;368:2385-94.

4. Peters S, Camidge DR, Shaw AT, Gadgeel S, Ahn JS, Kim DW, et al. Alectinib versus crizotinib in untreated alk-positive nonsmall-cell lung cancer. N Engl J Med. 2017;377:829-38.

5. Kim DW, Tiseo M, Ahn MJ, Reckamp KL, Hansen KH, Kim SW, et al. Brigatinib in patients with crizotinib-refractory anaplastic lymphoma kinase-positive non-small-cell lung cancer: a randomized, multicenter phase ii trial. J Clin Oncol. 2017;35:2490-8.

6. Patel M, Malhotra J, Jabbour SK. Examining eml4-alk variants in the clinical setting: the next frontier? J Thorac Dis. 2018;10: S4104-S4107.

7. Iyevleva AG, Raskin GA, Tiurin VI, Sokolenko AP, Mitiushkina $\mathrm{NV}$, Aleksakhina SN, et al. Novel alk fusion partners in lung cancer. Cancer Lett. 2015;362:116-21.

8. Soda M, Choi YL, Enomoto M, Takada S, Yamashita Y, Ishikawa S, et al. Identification of the transforming eml4-alk fusion gene in non-small-cell lung cancer. Nature. 2007;448:561-6.

9. Lindeman NI, Cagle PT, Aisner DL, Arcila ME, Beasley MB, Bernicker EH, et al. Updated molecular testing guideline for the selection of lung cancer patients for treatment with targeted tyrosine kinase inhibitors: guideline from the college of american pathologists, the international association for the study of lung cancer, and the association for molecular pathology. J Mol Diagn. 2018;20:129-59.

10. Teixido C, Karachaliou N, Peg V, Gimenez-Capitan A, Rosell R. Concordance of ihc, fish and rt-pcr for eml4-alk rearrangements. Transl Lung Cancer Res. 2014;3:70-74.

11. Li T, Maus MK, Desai SJ, Beckett LA, Stephens C, Huang E, et al. Large-scale screening and molecular characterization of eml4-alk fusion variants in archival non-small-cell lung cancer tumor specimens using quantitative reverse transcription polymerase chain reaction assays. J Thorac Oncol. 2014;9:18-25.

12. Piton N, Ruminy P, Gravet C, Marchand V, Colasse E, Lamy A, et al. Ligation-dependent rt-pcr: a new specific and low-cost technique to detect alk, ros, and ret rearrangements in lung adenocarcinoma. Lab Invest. 2018;98:371-9.

13. Reguart N, Teixido C, Gimenez-Capitan A, Pare L, Galvan P, Viteri S, et al. Identification of alk, ros1, and ret fusions by a multiplexed mrna-based assay in formalin-fixed, paraffinembedded samples from advanced non-small-cell lung cancer patients. Clin Chem. 2017;63:751-60.

14. Zheng Z, Liebers M, Zhelyazkova B, Cao Y, Panditi D, Lynch $\mathrm{KD}$, et al. Anchored multiplex pcr for targeted next-generation sequencing. Nat Med. 2014;20:1479-84.

15. Wang J, Mullighan CG, Easton J, Roberts S, Heatley SL, Ma J, et al. Crest maps somatic structural variation in cancer genomes with base-pair resolution. Nat Methods. 2011;8:652-4.

16. Yohe S, Thyagarajan B. Review of clinical next-generation sequencing. Arch Pathol Lab Med. 2017;141:1544-57.
17. Sussman RT, Oran AR, Paolillo C, Lieberman D, Morrissette JJD, Rosenbaum JN. Validation of a next-generation sequencing assay targeting rna for the multiplexed detection of fusion transcripts and oncogenic isoforms. Arch Pathol Lab Med. 2019.

18. Letovanec I, Finn S, Zygoura P, Smyth P, Soltermann A, Bubendorf L, et al. Evaluation of ngs and rt-per methods for alk rearrangement in european nsclc patients: Results from the european thoracic oncology platform lungscape project. J Thorac Oncol. 2018;13:413-25.

19. Lin C, Shi X, Yang S, Zhao J, He Q, Jin Y, et al. Comparison of alk detection by fish, ihc and ngs to predict benefit from crizotinib in advanced non-small-cell lung cancer. Lung Cancer. 2019;131: $62-68$.

20. Clave S, Rodon N, Pijuan L, Diaz O, Lorenzo M, Rocha P, et al. Next-generation sequencing for alk and ros 1 rearrangement detection in patients with non-small-cell lung cancer: implications of fish-positive patterns. Clin Lung Cancer. 2019;20:e421-e429.

21. Jennings LJ, Arcila ME, Corless C, Kamel-Reid S, Lubin IM, Pfeifer $\mathrm{J}$, et al. Guidelines for validation of next-generation sequencing-based oncology panels: a joint consensus recommendation of the association for molecular pathology and college of american pathologists. J Mol Diagn. 2017;19:341-65.

22. Presley CJTD, Soulos PR, Chiang AC, Longtine JA, Adelson KB, Herbst RS, et al. Association of broad-based genomic sequencing with survival among patients with advanced non-small cell lung cancer in the community oncology setting. JAMA. 2018;320: 469-77.

23. Reed GH, Wittwer CT. Sensitivity and specificity of singlenucleotide polymorphism scanning by high-resolution melting analysis. Clin Chem. 2004;50:1748-54.

24. Wittwer CT, Reed GH, Gundry CN, Vandersteen JG, Pryor RJ. High-resolution genotyping by amplicon melting analysis using lcgreen. Clin Chem. 2003;49:853-60.

25. Li M, Palais RA, Zhou L, Wittwer CT. Quantifying variant differences in DNA melting curves: effects of length, melting rate, and curve overlay. Anal Biochem. 2017;539:90-95.

26. Montgomery J, Wittwer CT, Kent JO, Zhou L. Scanning the cystic fibrosis transmembrane conductance regulator gene using highresolution DNA melting analysis. Clin Chem. 2007;53:1891-8.

27. Zhou L, Errigo RJ, Lu H, Poritz MA, Seipp MT, Wittwer CT. Snapback primer genotyping with saturating DNA dye and melting analysis. Clin Chem. 2008;54:1648-56.

28. Zhou L, Myers AN, Vandersteen JG, Wang L, Wittwer CT. Closed-tube genotyping with unlabeled oligonucleotide probes and a saturating DNA dye. Clin Chem. 2004;50:1328-35.

29. Distefano G, Caruso M, La Malfa S, Gentile A, Wu SB. High resolution melting analysis is a more sensitive and effective alternative to gel-based platforms in analysis of ssr-an example in citrus. PLoS One. 2012;7:e44202.

30. Enache EL, Sin A, Enache LS, Bancu L. Triplex high-resolution melting assay for the simultaneous assessment of ifnl3 rs12979860, abcb11 rs2287622, and rnf7 rs16851720 genotypes in chronic hepatitis c patients. J Mol Diagn. 2017;19:857-69.

31. Nicklas JA, Noreault-Conti T, Buel E. Development of a fast, simple profiling method for sample screening using high resolution melting (hrm) of strs. J Forensic Sci. 2012;57:478-88.

32. Zhou L, Palais RA, Paxton CN, Geiersbach KB, Wittwer CT. Copy number assessment by competitive per with limiting deoxynucleotide triphosphates and high-resolution melting. Clin Chem. 2015;61:724-33.

33. Li M, Liu L, Liu Z, Yue S, Zhou L, Zhang Q, et al. The status of kras mutations in patients with non-small cell lung cancers from mainland china. Oncol Rep. 2009;22:1013-20.

34. Zhao BX, Wang J, Song B, Wei H, Lv WP, Tian LM, et al. Establishment and biological characteristics of acquired gefitinib resistance in cell line nci-h1975/gefinitib-resistant with epidermal 
growth factor receptor $\mathrm{t} 790 \mathrm{~m}$ mutation. Mol Med Rep. 2015;11:2767-74.

35. Koivunen JP, Mermel C, Zejnullahu K, Murphy C, Lifshits E, Holmes AJ, et al. Eml4-alk fusion gene and efficacy of an alk kinase inhibitor in lung cancer. Clin Cancer Res. 2008;14: 4275-83.

36. Palais RA, Liew MA, Wittwer CT. Quantitative heteroduplex analysis for single nucleotide polymorphism genotyping. Anal Biochem. 2005;346:167-75.

37. Childress MA, Himmelberg SM, Chen H, Deng W, Davies MA, Lovly CM. Alk fusion partners impact response to alk inhibition: Differential effects on sensitivity, cellular phenotypes, and biochemical properties. Mol Cancer Res. 2018;16:1724-36.

38. Li Y, Zhang T, Zhang J, Li W, Yuan P, Xing P, et al. Response to crizotinib in advanced alk-rearranged non-small cell lung cancers with different alk-fusion variants. Lung Cancer. 2018;118: 128-33.

39. Noh KW, Lee MS, Lee SE, Song JY, Shin HT, Kim YJ, et al. Molecular breakdown: a comprehensive view of anaplastic lymphoma kinase (alk)-rearranged non-small cell lung cancer. J Pathol. 2017;243:307-19.

40. Woo CG, Seo S, Kim SW, Jang SJ, Park KS, Song JY, et al. Differential protein stability and clinical responses of eml4-alk fusion variants to various alk inhibitors in advanced alkrearranged non-small cell lung cancer. Ann Oncol. 2017;28: 791-7.

41. Lin JJ, Zhu VW, Yoda S, Yeap BY, Schrock AB, Dagogo-Jack I, et al. Impact of eml4-alk variant on resistance mechanisms and clinical outcomes in alk-positive lung cancer. J Clin Oncol. 2018;36:1199-206.
42. Yoshida T, Oya Y, Tanaka K, Shimizu J, Horio Y, Kuroda H, et al. Differential crizotinib response duration among alk fusion variants in alk-positive non-small-cell lung cancer. J Clin Oncol. 2016;34:3383-9.

43. Cha YJ, Kim HR, Shim HS. Clinical outcomes in alk-rearranged lung adenocarcinomas according to alk fusion variants. J Transl Med. 2016;14:296.

44. Montgomery JL, Sanford LN, Wittwer CT. High-resolution DNA melting analysis in clinical research and diagnostics. Expert Rev Mol Diagn. 2010;10:219-40.

45. Penzel R, Schirmacher P, Warth A. A novel eml4-alk variant: exon 6 of eml4 fused to exon 19 of alk. J Thorac Oncol. 2012;7:1198-9.

46. Zhang X, Zhang S, Yang X, Yang J, Zhou Q, Yin L, et al. Fusion of eml4 and alk is associated with development of lung adenocarcinomas lacking egfr and kras mutations and is correlated with alk expression. Mol Cancer. 2010;9:188.

47. Myrick JT, Pryor RJ, Palais RA, Ison SJ, Sanford L, Dwight ZL, et al. Integrated extreme real-time pcr and high-speed melting analysis in 52 to 87 seconds. Clin Chem. 2019;65:263-71.

48. Li M, Zhou L, Palais RA, Wittwer CT. Genotyping accuracy of high-resolution DNA melting instruments. Clin Chem. 2014;60:864-72.

49. Makrigiorgos GM. Extreme pcr meets high-speed melting: a step closer to molecular diagnostics "while you wait". Clin Chem. 2019;65:217-9.

50. Seipp MT, Pattison D, Durtschi JD, Jama M, Voelkerding KV, Wittwer CT. Quadruplex genotyping off5, f2, and mthfr variants in a single closed tube by high-resolution amplicon melting. Clin Chem. 2008;54:108-15. 